\title{
Marri, Mallee, Mulga: Pflanzenvielfalt Westaustraliens
}

\author{
Marianne Lauerer, Dean Nicolle, Malcolm French, \\ Annett Börner, Gregor Aas \& Ernst-Detlef Schulze
}

\begin{abstract}
Zusammenfassung
Westaustralien ist einer der „Hot Spots“ der Biodiversität der Erde. Es beherbergt über 10000 Pflanzenarten, darunter viele endemische. Von besonderer Bedeutung für diese Region sind Eukalypten, zu denen neben der bekannten Gattung Eucalyptus auch Corymbia gehört, die erst in jüngerer Zeit als eigene Gattung von Eucalyptus abgetrennt wurde. Dargestellt werden verschiedene von Eukalypten dominierte Vegetationstypen Westaustraliens, ferner die küstennahen Banksia-Heidegebüsche im Südwesten, Acacia-Buschländer und die halbimmergrünen Trockenwälder und Savannen der Kimberleys.
\end{abstract}

\begin{abstract}
Western Australia is one of the 'hot spots' of global biodiversity with more than 10000 plant species, many of which are endemic to this region. In the minds of many people Australia is associated with eucalypts, including species of the genera Eucalyptus and Corymbia, which was recently separated from Eucalyptus. Different vegetation types of Western Australia dominated by eucalypts are described, also Banksia-heathlands, Acacia shrublands and tropical dry forests and savannahs from the Kimberley.
\end{abstract}

\section{Einführung}

Aus europäischer Sicht erscheint Australien vor allem als Kontinent der endlosen, unberührten Landschaften. Wenn auch nicht für ganz Australien, so trifft das doch für Westaustralien zu, einem der am geringsten vom Menschen besiedelten Teile dieses Kontinents, wo in den meisten Regionen weniger als 0,1 Einwohner pro $\mathrm{km}^{2}$ leben (Braun et al. 2010). Im Juli und August 2010 erkundeten wir im Rahmen einer botanischen Exkursions- und Sammelreise Teile Westaustraliens (Abb. 1). Ziel war es, Blattproben von Akazien für ${ }^{15} \mathrm{~N}$-Analysen zu sammeln.

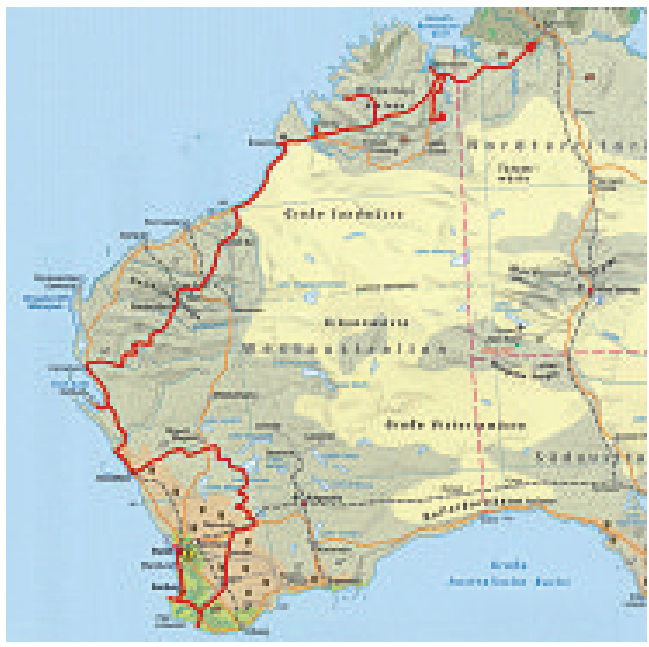

Der Anteil an stabilen ${ }^{15} \mathrm{~N}$-Isotopen im Pflanzenmaterial gibt Aufschluss über das Maß der Stickstoffversorgung über Knöllchenbakterien und trägt zur Beantwortung der Fragestellung bei, ob vom mediterranen Winterregengebiet im Süden bis zum tropischen Sommerregengebiet im Norden die Akazien unterschiedlich stark von einer Stickstoffversorgung über Knöllchenbakterien profitieren. Zudem suchten wir Standorte von ausgewählten Eukalypten auf, um detaillierte Informationen über die Verbreitung dieser Arten zu bekommen und Untersuchungen über die Rolle der Artenvielfalt bei der Anpassung von Pflanzen an Trockenheit zu ergänzen (Schulze et al. 2006 a, b, Turner et al. 2010). Last but not least sammelten wir Material zur Kultur von (Eukalyptus-)Arten im Currency Creek Arboretum in Adelaide und im Ökologisch-Botanischen Garten der Universität Bayreuth.

\section{Naturraum, Geologie und Klima}

Australien lässt sich in drei Großlandschaften gliedern: das Westaustralische Tafelland, die Mittelaustralische Senke und die Ostaustrali-

Abb. 1: Westliches Australien. Rot eingetragen ist die Route der Exkursion im Juli/August 2010 (Kartengrundlage aus Praxis Geographie 2010). 

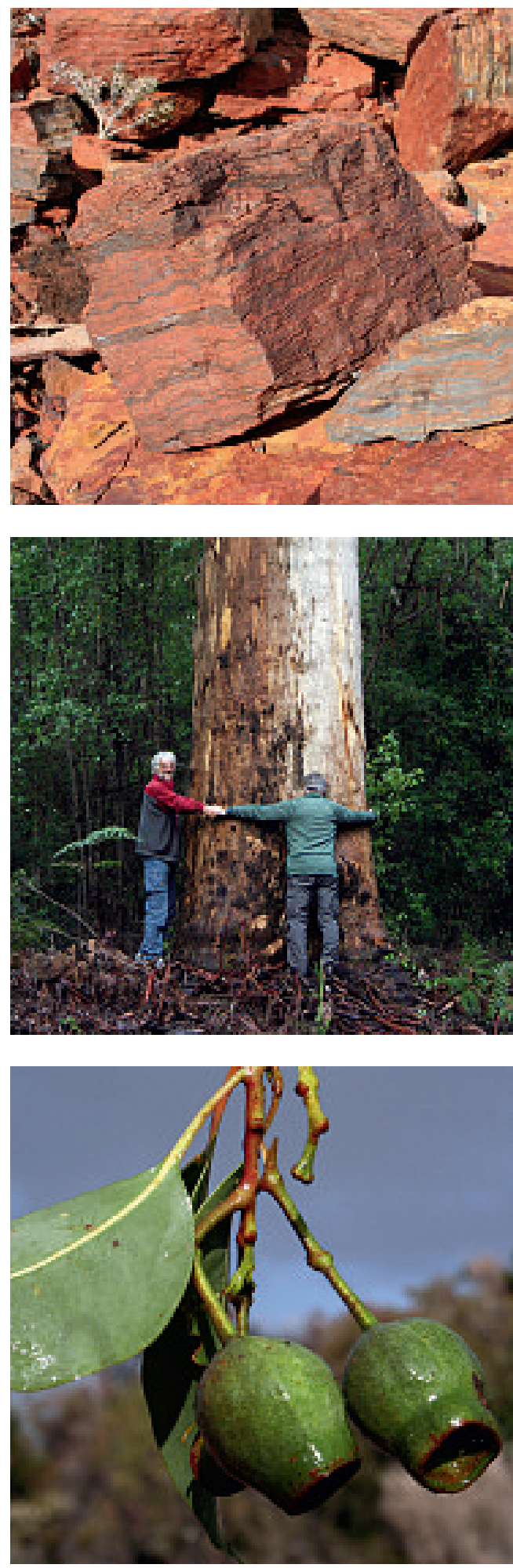

schen Randgebirge. Der Bundesstaat Westaustralien liegt vollständig im westlichen Tafelland, das mittlere Höhen von $300-400(800) \mathrm{m}$ aufweist, von großen Salzseen geprägt ist und aus der einige wenige Gebirgszüge emporragen wie die Hamersley oder Barlee Range. Geologisch besteht Westaustralien hauptsächlich aus präkambrischen Graniten und Gneisen, die bis zu 3,2 Milliarden Jahre alt sind. Vor mehr als 500 Millionen Jahren zerbrach das präkambrische Schild in große Blöcke, senkte sich ab und wurde lokal mit Sedimenten verfüllt.

Zeugen für das erdgeschichtlich hohe Alter sind die in Westaustralien vorkommenden Stromatolithen und Bändereisenerze (Banded Iron). Vor ungefähr 3,5 Milliarden Jahren gab es erste Spuren von Leben im Meer: Cyanobakterien (Blaualgen) waren die ersten Organismen, die mittels Photosynthese für Sauerstoff auf der Erde sorgten und damit die Grundlage unseres Lebens schafften. In Gemeinschaft lebend, bildeten sie durch Einfangen und Bindung von Sedimentpartikeln und/oder durch Fällung gelöster Stoffe (Kalk) über Millionen von Jahren biogene Sedimentgesteine, die sogenannten Stromatolithen, Leitfossilien des Präkambriums und die ältesten Fossilien überhaupt. Heute gibt es Stromatolithen nur noch an wenigen Stellen weltweit, eine davon in Hamelin Pool in Westaustralien (1956 entdeckt, inzwischen UNESCO Weltnaturerbe). Überlebt haben diese Cyanobakterien aufgrund des hohen Salzgehaltes im Wasser in der Lagune von Hamelin Pool, da unter diesen lebensfeindlichen Bedingungen ihre natürlichen Feinde fehlen.

Bändereisenerze sind im Meer entstandene Sedimentgesteine aus dem frühen Proterozoi-

Abb. 2 (oben): Bändereisenerz, ein über drei Milliarden Jahre altes, eisenhaltiges Sedimentgestein, das für Westaustralien typisch ist (Mt. Sheila, nahe Tom Price).

Abb. 3 (Mitte): Stamm eines Eucalyptus diversicolor (Karri) im Warren Nationalpark im äußersten Südwesten Australiens.

Abb. 4 (unten): Früchte von Corymbia calophylla (Marri), einem Eukalyptus aus den Wäldern des feuchten Südwestens. 
kum, deren Entstehung eng mit der ersten Sauerstoffbildung zusammenhängt. In charakteristischer Schichtung von einigen Millimetern bis Zentimetern Mächtigkeit wechseln sich eisenhaltige Schichten mit mikrokristallinen Silikatlagen (Jaspis) ab (Abb. 2). Es wird angenommen, dass das Eisen durch das Ausströmen eisenhaltiger Lösungen am Mittelozeanischen Rücken und entlang von Tiefseegräben in das Meerwasser gelangte. Der von Cyanobakterien freigesetzte Sauerstoff wurde im Proterozoikum zunächst chemisch im Meer gebunden, oxidierte das gelöste Eisen zu wasserunlöslichen Verbindungen, die schließlich in Bändereisenerze abgelagert wurden. In jüngster Zeit werden Bändereisenerze in großem Stil in der Pilbara-Region abgebaut. Australien ist weltweit eines der drei bedeutendsten Förderländer für Eisenerz, der Großteil wird nach China exportiert (Thielke 2010).

\section{Pflanzenwelt}

Die australische Flora gilt als einzigartig, einerseits aufgrund der hohen Zahl an Arten und Endemiten (ca. 20000 Blütenpflanzen, LüPNITZ 1998) andererseits, weil nur zwei, allerdings extrem diverse Pflanzengattungen die Flora des Kontinents prägen: Eukalypten und Akazien (BEADle 1981). Westaustralien beherbergt über 10000 Arten höherer Pflanzen aus über $200 \mathrm{Fa}-$ milien, deren artenreichsten die Fabaceae, Myrtaceae, Proteaceae, Poaceae und Asteraceae sind. Ihr Anteil an Endemiten beträgt 50 - 80\% (Western Australia Flora 2010). Als Gründe für den hohen Artenreichtum wird einerseits die Habitatkonstanz über lange Zeiträume angeführt, zum andern aber Feuer als ökologischer Faktor, der neue Lebensräume schafft, in denen sich mutierte Merkmale etablieren können.

Abb. 5 (oben): Bestand mit Eucalyptus salmonophloia (Salmon Gum), der auf roten Ton- und Sandböden des südlichen Westaustralien vorkommt.

Abb. 6 (Mitte): Ein typischer mehrstämmiger MalleeEukalyptus mit Lignotuber (Eucalyptus ebbanoensis).

Abb. 7 (unten): Mallee-Eukalypten wie Corymbia lenziana können nach einem Brand aus den schlafenden Knospen des Lignotubers wieder austreiben.
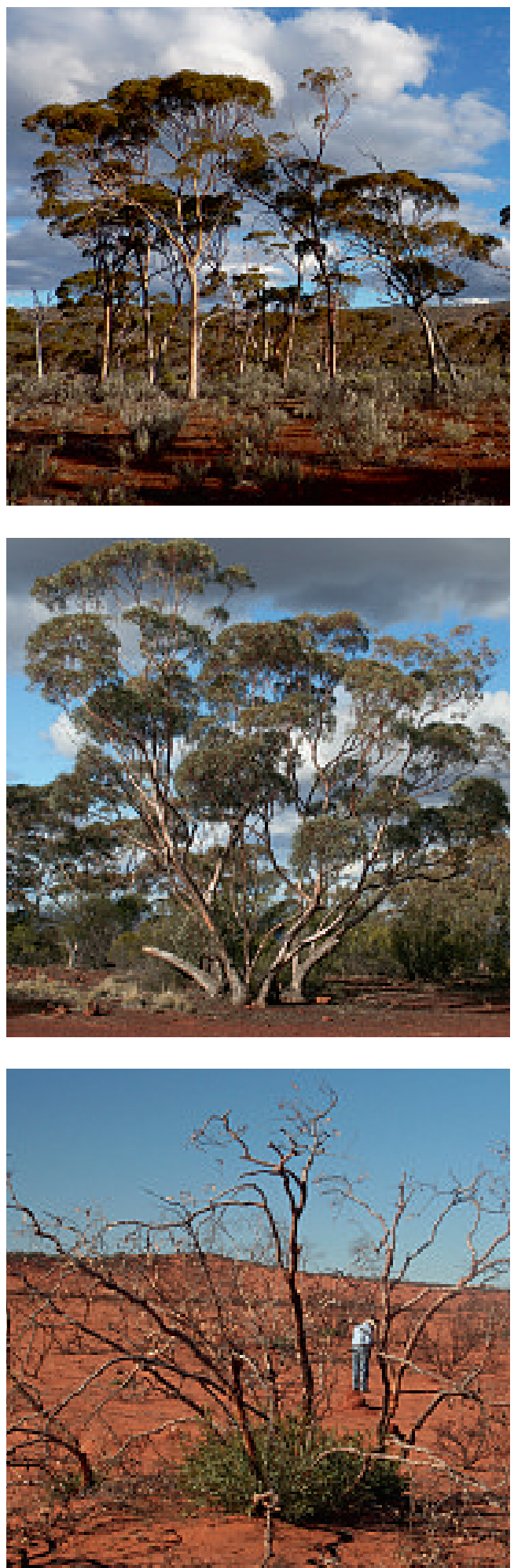


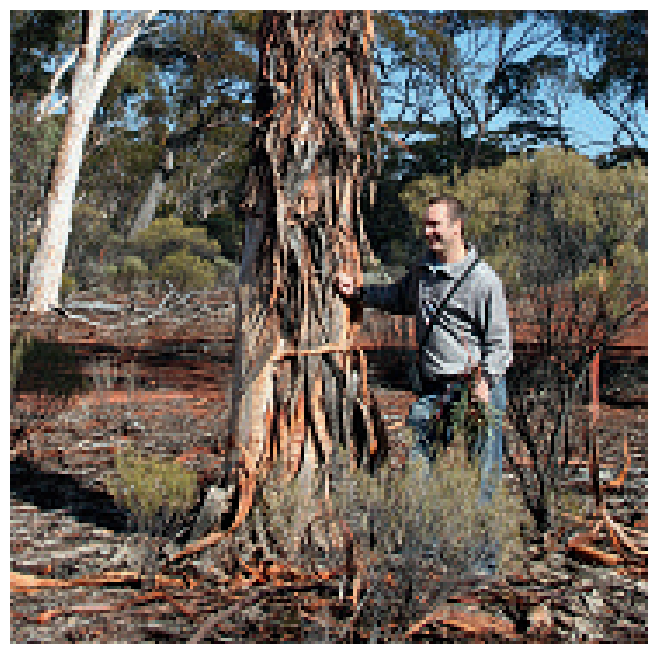

Im Folgenden werden fünf wichtige Vegetationstypen und einige charakteristische Taxa vorgestellt.

\subsection{Corymbia: Eukalypten, aber kein Eucalyptus}

Kein anderer Kontinent hat einen so stark von einer einzigen Gattung dominierten Baumbestand wie Australien, und durch keine andere Pflanzengattung wird die Eigentümlichkeit der australischen Flora besser charakterisiert als durch Eucalyptus. Es gibt nur wenige Lebensräume in Australien ohne Eukalypten, und nur etwa 15 der insgesamt über 900 Arten der Gattung kommen auch außerhalb Australiens und nur zwei nicht in Australien vor (Hill 1991, Nicolle 1997, Brooker \& Kleinig 2006).

Eine erste umfassende Klassifikation der Eukalypten stammt aus der ersten Hälfte des vorigen Jahrhunderts (Blakely 1934), in der über 600 Taxa beschrieben sind. 1971 gliedern PrYOR \& JoHnson die Gattung in sieben Subgenera und trennen Angophora als eigene Gattung ab. Auf der Grundlage morphologischer und molekularer Daten schlagen Hill \& Johnson (1995) dann auch die Abtrennung von Corymbia (Bloodwoods, Ghost Gums, Spotted Gums) als Schwestergruppe zu Angophora vor. Auch wenn Corymbia als eigenständige Gattung noch nicht überall Eingang gefunden hat (BROOKER 2000, Brooker \& Kleinig 2006), so gilt sie mittlerweile als akzeptiert (Plant List 2010). Sie umfasst über 100 Arten, darunter auch E. gummifera, den ersten von BAnks und Solander 1770 in der Botany Bay gesammelten Eukalyptus, nun Corymbia gummifera (Gaertn.) Hill \& Johnson. Auch einige in Europa häufiger kultivierte Arten gehören zu Corymbia (Tab. 1). Umgangssprachlich bleiben alle weiterhin $\mathrm{Eu}-$ kalypten (engl. eucalypts).

Angophora unterscheidet sich von Eucalyptus s.str. durch das fehlende Operculum und die meist auch im Alter gegenständig angeordneten

\begin{tabular}{|c|c|c|}
\hline Alter Name (Syn.) & Trivialname & Gültiger Name \\
\hline Eucalyptus calophylla & Marri & $\begin{array}{l}\text { Corymbia calophylla } \\
\text { (R. BR. ex LindL.) HiLl \& JoHnson }\end{array}$ \\
\hline Eucalyptus citriodora & Lemon Scented Gum & Corymbia citriodora (Ноок.) Hill \& JoHnson \\
\hline Eucalyptus eximia & Yellow Bloodwood & Corymbia eximia (Schauer) Hill \& Johnson \\
\hline Eucalyptus ficifolia & Red Flowering Gum & $\begin{array}{l}\text { Corymbia ficifolia } \\
\text { (F. Muell.) Hill \& JoHnson }\end{array}$ \\
\hline Eucalyptus gummifera & Red Bloodwood & $\begin{array}{l}\text { Corymbia gummifera } \\
\text { (GAERTN.) Hill \& JoHnson }\end{array}$ \\
\hline Eucalyptus maculata & Spotted Gum & Corymbia maculata (Ноок.) Hill \& Johnson \\
\hline Eucalyptus ptychocarpa & Swamp Bloodwood & $\begin{array}{l}\text { Corymbia ptychocarpa } \\
\text { (F. Muell.) Hill \& JoHnson }\end{array}$ \\
\hline
\end{tabular}

Tab. 1: Auswahl an Eukalypten, die zur Gattung Corymbia gehören (Hill \& Johnson 1995, Plant List 2010). 
Blätter. Die Identifizierung von Corymbia ist schwieriger und oft nur durch Merkmalskombinationen möglich. Der Blütenstand von Corymbia ist eine zusammengesetzte, endständige Dolde, die Filamente sind in der Knospe nicht "gebeugt", die Antheren öffnen sich durch parallele Schlitze und die Borke ist glatt oder blättert in kleinen polygonen Plättchen ab (HILl \& JoHNSON 1995).

\subsection{Die Riesen Westaustraliens: Karri und Jarrah}

Ganz im Südwesten Australiens, in Gebieten mit Mediterranklima und mehr als $1000 \mathrm{~mm}$ Niederschlag pro Jahr (vorwiegend im Winter), dominieren von Natur aus feuchte Hartlaubwälder. In dieser Region, die zu den Hot Spots der Biodiversität auf der Erde zählt (LüPNITZ 1998, 2003, Western Australia Flora 2011), kommen etwa 6000 Pflanzenarten vor, von denen rund $75 \%$ endemisch sind. Aufgrund der Küsten im Süden und Westen und der Gebirgszüge als natürliche Barriere im Nordosten zum abrupt trockener werdenden Landesinnern ist diese Region floristisch isoliert. Im Unterschied $\mathrm{zu}$ anderen Mediterrangebieten der Erde herrschen hier stattliche Wälder vor, deren Baumschicht nahezu ausschließlich Eukalypten bilden. Fünf Arten sind charakteristisch: Karri (Eucalyptus diversicolor, Abb. 3) und Jarrah (E. marginata) als die häufigsten sowie Marri (Corymbia calophylla, Abb. 4), Wandoo (E. wandoo) und Tuart (E. gomphocephala, BEADLE 1981, Diels 1906).

Die Verbreitung des Karri beschränkt sich auf den äußersten Südwesten mit über $1200 \mathrm{~mm}$ Niederschlag und tiefgründigen Böden (Western Australia Flora 2010, Fukarek et al. 1995).

Abb. 8 (Seite 34): Stark abblätternde Borke von Eucalyptus vittata, einer Art, die erst 2009 von Dean Nicolle (im Bild) neu beschrieben wurde.

Abb. 9 (oben rechts): Blüte und Blütenknospe mit geripptem Operculum von Eucalyptus kingsmillii.

Abb. 10 (unten rechts): Blüten von Eucalyptus miniata, einem spektakulär blühenden Eukalyptus aus den Kimberleys.
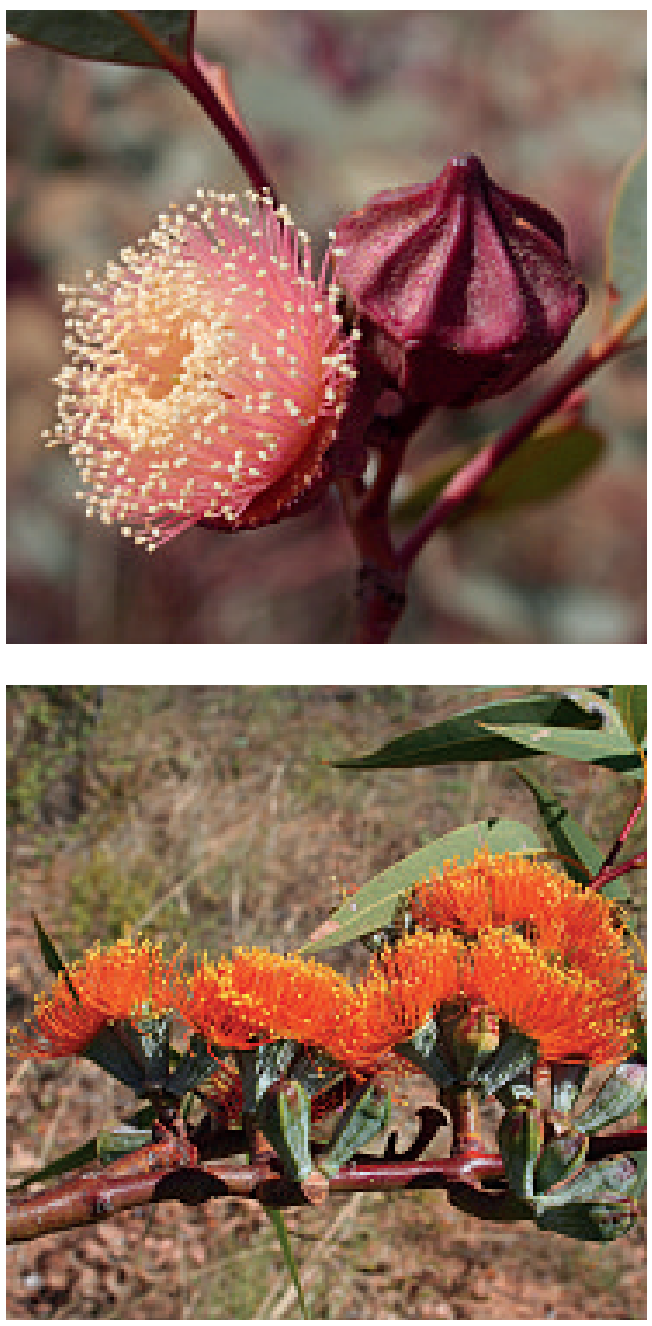

Er ist mit Höhen von 40-70 m der größte aller westaustralischen Bäume und gehört mit maximal $90 \mathrm{~m} \mathrm{zu}$ den höchsten Bäumen der Erde. Beeindruckende Exemplare kommen im Warren Nationalpark vor. Der Name E. diversicolor geht auf die Farbe der Borke zurück, die sich in großen Fetzen vom Stamm löst und gelblichweißlich-rötliche Flecken hinterlässt (Abb. 3). Schon in den 1930er- und 1940er-Jahren machte man sich die stattliche Höhe und den geraden Wuchs zu Nutze und legte in den Kronen von Karri-Bäumen Aussichtsplattformen an, um Buschbrände beizeiten erkennen zu können. Mit $63 \mathrm{~m}$ ist eine solche Plattform auf dem Glouchester Tree nahe Pemberton die höchste 

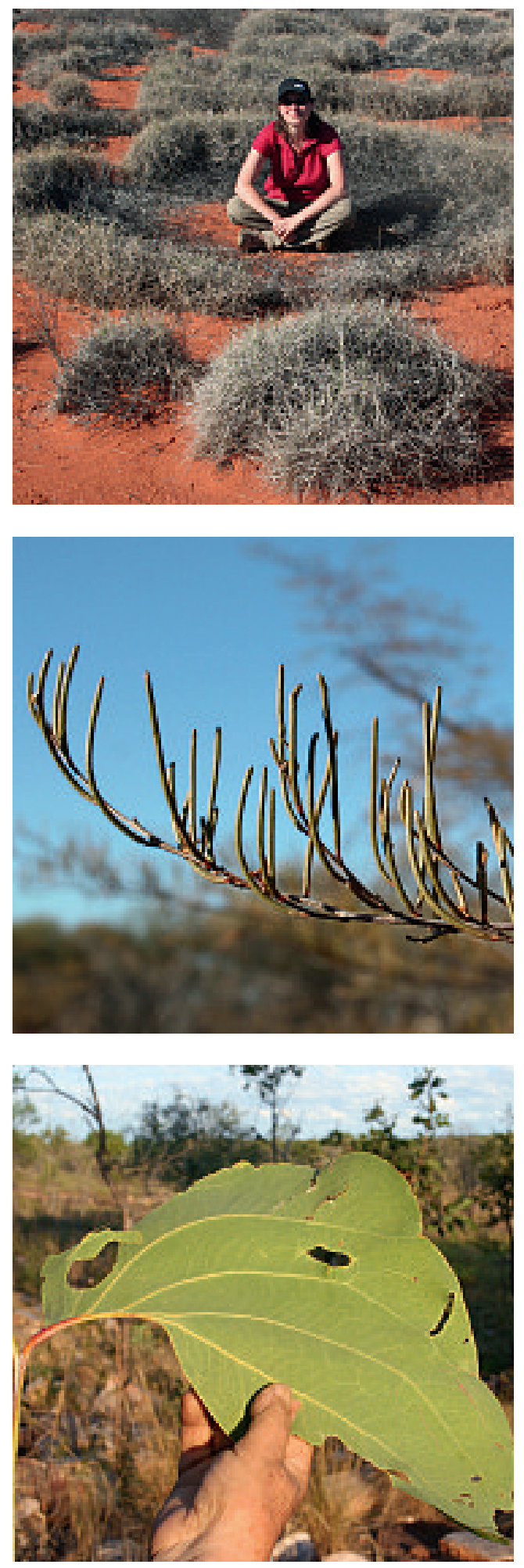

der Welt und bietet auch Besuchern eine spektakuläre Aussicht über die Karriwälder.

Mit dem Karri oft vergesellschaftet ist der nahe verwandte Jarrah (Eucalyptus marginata). Er kommt mit weniger Niederschlägen aus, wird nur bis $40 \mathrm{~m}$ hoch. Seine Borke löst sich in Längsstreifen und die Blätter haben entlang des Blattrandes eine hell durchscheinende Blattaderung („marginata“). Die Wurzeln reichen bis in eine Tiefe von $40 \mathrm{~m}$, sodass sich Jarrahs während der trockenen Sommer Wasser aus großer Tiefe holen können. Die Hölzer von Jarrah und Karri gehören zu den wertvollsten Harthölzern Australiens.

\subsection{Mallee: Vielfalt auf großer Fläche}

Mit zunehmender Entfernung von der Küste zum Landesinnern hin wird das Klima rasch arider. In einem Vegetationsgürtel, der im südlichen und westlichen Australien die zentralen Wüstengebiete umgibt, dominieren niederwüchsige, nur acht Meter hohe und oft mehrstämmige Eucalyptus-Arten (BEADLE 1981, Abb. 6). Typisch für diese sogenannten MalleeEukalypten ist ihr Lignotuber, eine Verdickung an der Stammbasis mit vielen schlafenden Knospen. Der Begriff Mallee stammt aus der Sprache der Aborigines und wird auch für den Vegetationstyp verwendet, in dem MalleeEukalypten vorkommen (BEAdLE 1981). Im Unterwuchs der Mallee sind Atriplex- und Triodia-Arten (Spinifex) häufig, aber auch xeromorphe, strauchartige Proteaceae und Myrtaceae. Große Teile der südlichen Mallee-Region werden als Rinderweide genutzt.

Auf den ersten Blick wirkt die ausgedehnte Mallee-Region recht einheitlich, doch ist ihre Struktur und Artenzusammensetzung ausgesprochen vielfältig. Mehrere hundert Euka-

Abb. 11 (oben): Bestand von Spinifex (Triodia sp., Poaceae).

Abb. 12 (Mitte): Phyllodien von Acacia aneura, die als Anpassung an die Trockenheit nadelartig ausgebildet sind.

Abb. 13 (unten): Wegen seiner riesigen Phyllodien wird Acacia dunnii als Elephant Ear Wattle bezeichnet. Der kleine Baum kommt in den Kimberleys vor. 
lypten kommen hier vor, von denen die meisten nur ein kleines Areal besiedeln, obwohl offensichtliche Ausbreitungsbarrieren fehlen. Diese Einnischung der Arten innerhalb der Mallee-Region beruht auf edaphischen, klimatischen und hygrischen Gegebenheiten. Die Niederschläge betragen im langjährigen Mittel nur 200-400 mm im Jahr, wobei oft mehrere Jahre lang gar kein Regen fällt umso erstaunlicher, dass hier Bäume wachsen können! Die hier vorkommenden Bäume vertragen Salz, differenzieren ihr Wurzelsystem und regulieren ihre Blattdicke (ScHulze et al. 2006 a, b).

Feuer ist im Mallee ein wichtiger ökologischer Faktor. Etwa alle 20 Jahre treten natürliche Brände auf, gefördert durch die abblätternde Borke vieler Eukalypten (Abb. 8), die um die Stämme eine dicke, leicht entzündliche Streuschicht bildet. Mallee-Eukalypten sind gut an Feuer angepasst, streuen ihre Samen bevorzugt nach Bränden aus und können sich rasch aus schlafenden Knospen des Lignotubers regenerieren (Abb. 7, Williams \& WoinARski 1997). Die Stämme sind selten älter als ein paar Jahrzehnte, ein Lignotuber aber kann mehrere Generationen an Stämmen hervorbringen, Hunderte von Jahren alt werden und mehrere Meter Durchmesser erreichen. Der strauchförmige Wuchs des Mallee folgt dem Prinzip, dass das Absterben von Einzelsprossen nicht das Leben der Gesamtpflanze gefährdet (survival through dieback, Evenari et al. 1982).

\subsection{Acacia: die artenreichste Gattung in Australien}

Die artenreichste Gattung in Australien - noch vor den Eukalypten - sind mit etwa 950 Arten und über 1200 Sippen die Akazien (LüpNitz

Abb. 14 (oben): Drosera cf. ordensis nahe Derby Airport in einer Baobab-Savanne.

Abb. 15 (Mitte): Blütenstand von Banksia menziesii.

Abb. 16 (unten): Blütenstand von Grevillea cf. dielsiana im Kalbarri National Park.
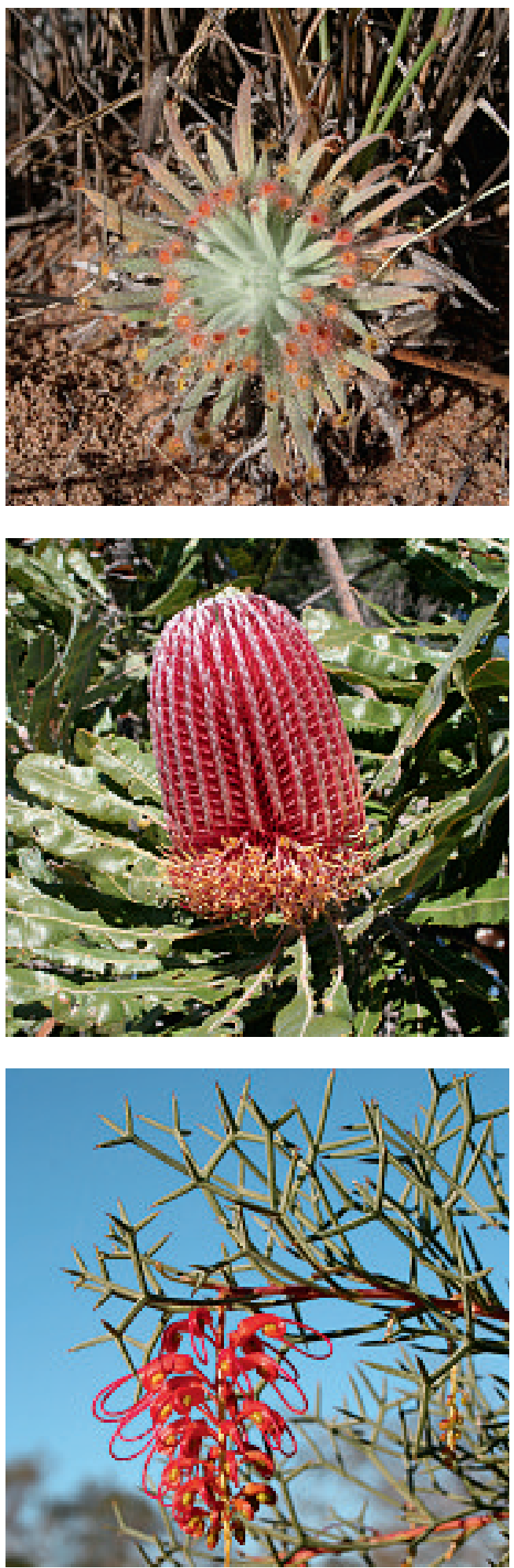


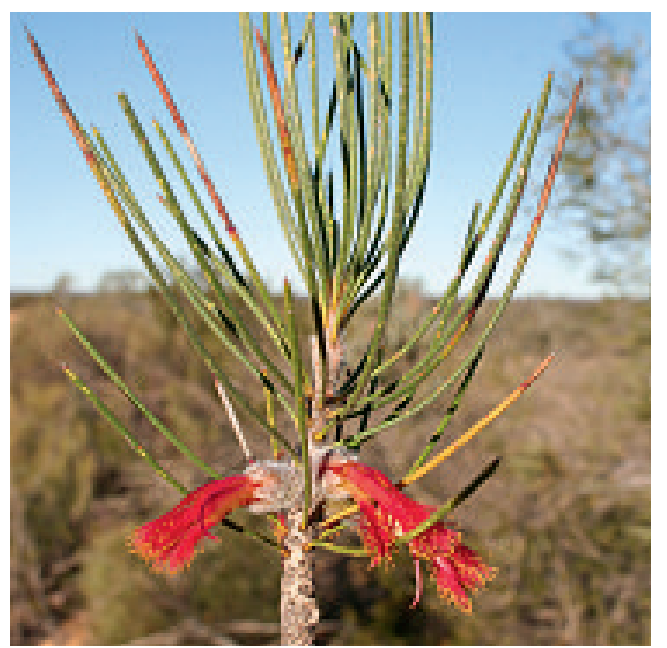

1998). Sie dominieren vor allem in den trockeneren Gebieten, wo Eukalyptus an Bedeutung verliert, sind selten höher als fünf bis sieben Meter und wachsen meist strauchförmig in lichten Beständen. Die meisten australischen Akazien haben im Unterschied zu den afrikanischen und südamerikanischen keine Fiederblätter, sondern Phyllodien, die sich aus dem verbreiterten Blattstiel, oft unter Einbeziehung der Blattrhachis, bilden und an Stelle der Blattspreite die Photosynthese übernehmen. Nur die Primärblätter der Keimlinge sind stets gefiedert. Die Phyllodien sind vielgestaltig, können riesige „Blätter“ bilden wie bei der Elephant Ear Wattle (Acacia dunnii, Abb. 13) aus den Kimberleys oder können nadelartig sein, wie bei $A$. aneura (Abb. 12). Acacia-Phyllodien haben stets eine kleine Drüse an der keilförmigen Basis oder am Stiel und sind dadurch von Blättern anderer Gattungen zu unterscheiden. Das Holz vieler Akazien wird wegen seiner Widerstandsfähigkeit geschätzt und z.B. zur Herstellung von Zaunpfählen, Stöcken, Speeren und Bumerangs genutzt. Akazien sind dekorative Pflanzen für Botanische Gärten. Vor der Aussaat sollten die Samen mit kochend heißem Wasser überbrüht und 12-24 Stunden eingeweicht werden. Die Samen beginnen zu quellen und keimen dann nach der Aussaat rasch innerhalb von 7-20 Tagen (Elliot \& Jones 1985, Brock 2007).

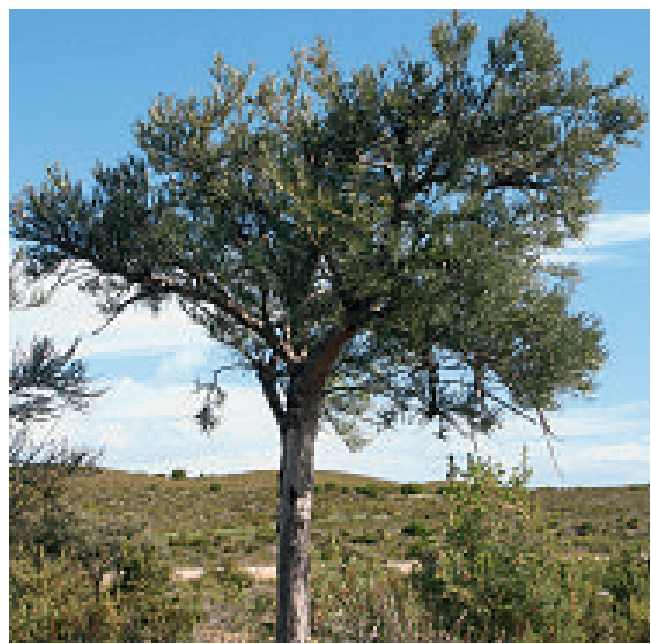

\subsection{Sandheiden mit Banksien und anderen floristischen Besonderheiten}

Auf sandigen Böden wird Eucalyptus durch die australische Heidevegetation ersetzt, die sehr artenreich ist und einen mehr oder weniger breiten Gürtel vor allem entlang der Küsten Südwestaustraliens bildet. Der erste Eindruck dieser meist graugrünen Sandheiden mag als eintönig empfunden werden (DiELs 1906), bei näherem Hinsehen aber überrascht die floristische Vielfalt der Pflanzen, die sowohl an extremen Nährstoff- wie auch an Wassermangel angepasst sind. Häufig sind Arten der Gattungen Banksia (Abb. 15), Grevillea (Abb. 16), Hakea, Conospermum und andere Proteaceae, Anigozanthos (Haemadoraceae, Kangaroo Paw), Acacia, Eucalyptus (z. B. E. gittinsii) und weitere Myrtaceae wie Calothamnus (Abb. 17).

Banksien (Proteaceae) sind die vielleicht spektakulärsten Blütenpflanzen Australiens. In der australischen Bucht, der Botany Bay, sam-

Abb. 17 (oben links): Blüten von Calothamnus cf. chrysanthereus (Myrtaceae) im Kalbarri National Park.

Abb. 18 (oben rechts): Nuytsia floribunda, ein baumförmiger Halbschmarotzer aus dem südwestlichen Westaustralien.

Abb. 19 (Seite 39): Savanne mit Adansonia gregorii, einem Flaschenbaum, der typisch für die Sommerregengebiete Nordwest-Australiens ist. 
melte Sir Joseph Banks (1743-1820), der als Botaniker James CooK auf dessen erster Weltumsegelung begleitete, um 1770 vier Vertreter der bis dahin der Wissenschaft unbekannten Gattung, die später zu seinen Ehren den $\mathrm{Na}$ men Banksia erhielt. Linne's Sohn war von dieser Aufsammlung so begeistert, dass er vorschlug, den ganzen Kontinent nach Sir Banks zu benennen. Wäre man diesem Vorschlag gefolgt, hieße der australische Kontinent heute vielleicht „Banksien“!

Heute kennt man an die 100 Banksia-Arten (TAylor \& Hopper 1991), allein im Südwesten Australiens kommen 60 vor. Außer Banksia dentata, die auch in New Guinea heimisch ist, sind alle Arten in Australien endemisch (ELliot \& Jones 1985). Die meisten kommen in küstennahen Gebieten auf sandigen Böden oder sogar auf reinem Sand vor und sind Sträucher von wenigen Metern Höhe (TAYlor \& Hopper 1991). Die Blütenstände enthalten nicht selten Tausende von Einzelblüten und sind oft sehr dekorativ gelb, orange oder rot gefärbt. Ihre charakteristischen Fruchtstände aus verholzten Balgfrüchten öffnen sich bei vielen Arten erst nach einem Buschfeuer und entlassen dann ihre beiden flugfähigen Samen.

Eine floristische Besonderheit, die nur in den Sandheiden ganz im Südwesten von Westaustralien vorkommt, ist Nuytsia floribunda (Loranthaceae, Western Australian Christmas Tree, Abb. 18). Sie wird ein bis zu zehn Meter hoher Baum und fällt daher in der niedrigeren Heidevegetation auf. Nuytsia ist einer der wenigen baumförmigen Halbschmarotzer der Erde, der Photosynthese betreibt, sich aber Wasser und Nährstoffe von Wirtspflanzen holt (PATE \& DiXon I982, Elliot \& Jones I997, Calladine \& Pate 2000). Untersuchungen zeigten, dass Nuytsia sich von einer ganzen Reihe von Wirtspflanzen ernähren kann, wie Banksien und anderen Proteaceen, Myrtaceen (u. a. Eukalypten, Melaleuca und Kunzea) sowie Xanthorrhoea preissii, wenngleich diese Beziehungen nicht obligat sind und Nuytsia in den ersten Lebensjahren auch ohne Wirt gut wachsen kann (CALladine et al. 2000).

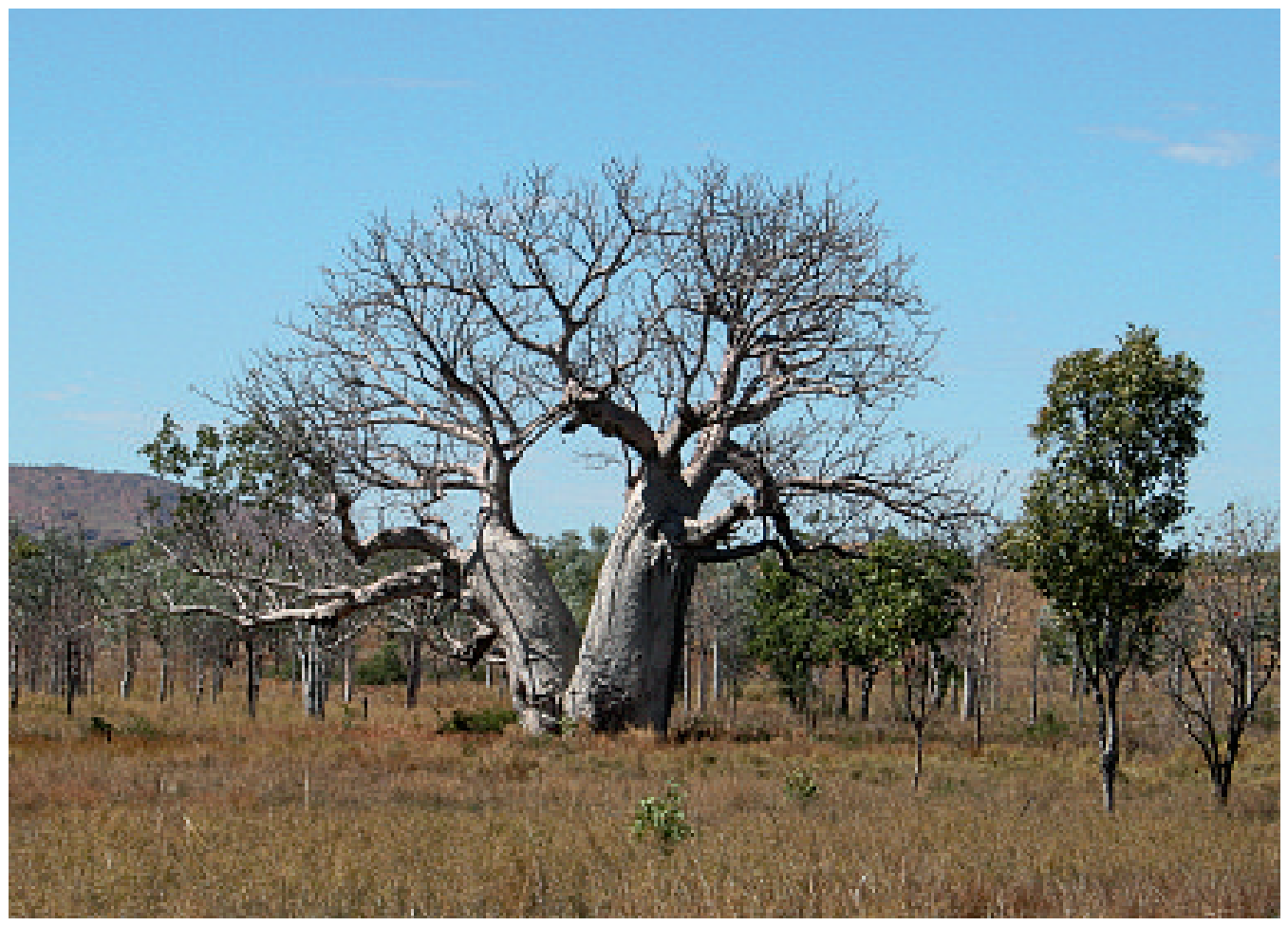




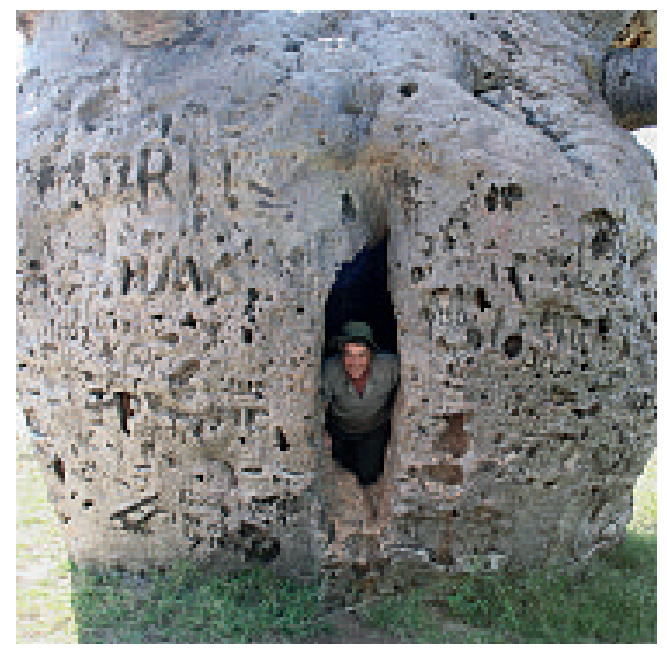

Nährstoffarme Sande, auf denen Stickstoff und Phosphor nur unzureichend zur Verfügung stehen, sind ideale Standorte für Insekten fangende Pflanzen, die sich durch Tierfang Stickstoff erschließen (Schulze et al. 1991). Allein 70 Drosera-Arten kommen in Australien vor (LüpNITZ 2003), wobei Westaustralien als das Zentrum der Diversität karnivorer Pflanzen gilt (Lowrie 1998). Außerhalb Australiens sind Drosera-Arten vor allem in Sümpfen und Mooren verbreitet. Die meisten australischen Arten aber wachsen auf reinem Sand und in ariden Klimaten (Lowrie 1987). Die trockenen Monate überdauern viele von ihnen mittels unterirdischer Knollen (modifizierte Stolone) einige Zentimeter (bis $\mathrm{zu} 60 \mathrm{~cm}$ ) tief im Boden (Low RIE 1987). Einige Drosera-Arten sind Kletterer wie $D$. macrantha oder $D$. menziesii, andere Rosetten wie D. ordensis (Abb. 14) aus den tropischen Regionen Westaustraliens. Die starke Behaarung der Blätter schützt die junge Pflanze in den Sommermonaten vor Austrocknung und dient der Verbesserung der Wasserversorgung, indem Tau durch Kondensation ausgekämmt und der Pflanze verfügbar wird (Lowrie 1998).

\subsection{Baobab: mehr breit als hoch}

In den Sommerregengebieten der Kimberleys (nördliches Westaustralien) prägen halbimmergrüne Trockenwälder und Savannen, die von Baobabs (Adansonia gregorii) durchsetzt sind,

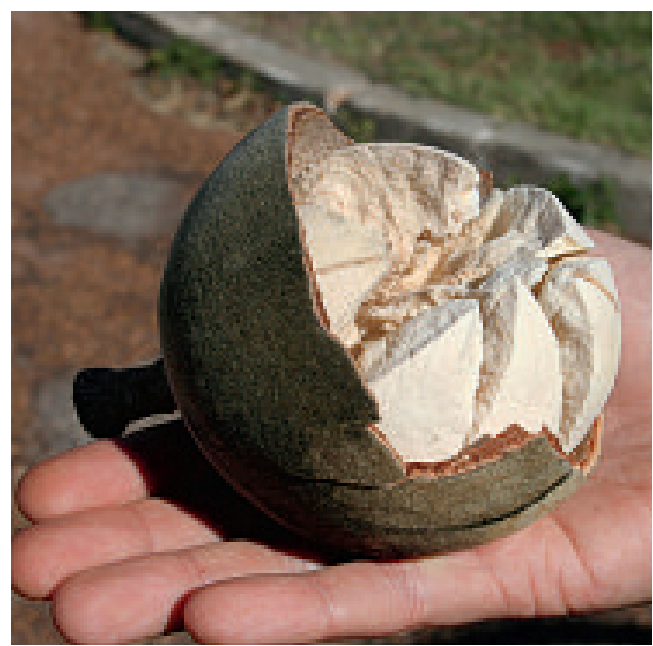

das Landschaftsbild. Der australische Baobab hat seine nächsten Verwandten in Madagaskar (sechs Arten) und Afrika (eine Art) und ist der einzige Vertreter dieser Gattung in Australien. Er wird nur $15 \mathrm{~m}$ hoch, aber bis zu $20 \mathrm{~m}$ dick und bildet typischerweise einen flaschenartigen Stamm zur Wasserspeicherung aus (Abb. 19). Er ist in den trockenen Wintermonaten laublos und blüht und fruchtet im Sommer. Die großen, behaarten Früchte enthalten zahlreiche Samen, die von einer mehligen Pulpa (Fruchtmark) umgeben sind (Abb. 21). Die Aborigines haben dieses Fruchtmark, das reich an Vitamin $C$ ist, gegessen, aber auch die jungen Blätter medizinisch oder in Salaten verwendet (BROCK 2007). Unabhängig von diesen traditionellen Nutzungen wird der Baobab in jüngster Zeit in den Kimberleys kommerziell angebaut. Nach der Aussaat bilden sich in wenigen Wochen rübenartige Wurzeln, die geerntet werden, wenn sie die Größe einer Karotte haben. Auf lokalen Märkten (z.B. in Kununurra) werden sie in Bündeln verkauft und können als Gemüse zubereitet werden.

Abb. 20 (links): Große und innen hohle Baobabs wurden als "Gefängnis" genutzt, wie dieser Adansonia gregorii südlich von Derby.

Abb. 21 (rechts): Geöffnete Frucht von Adansonia gregorii. 
Im hohen Alter sind Baobab-Stämme innen oft hohl. Ein derartiger stattlicher Baum steht nahe Derby an der Gibb River Road. Er soll 1500 Jahre alt sein, hat einen Umfang von über 14 Metern und eine Öffnung, über die man den Innenraum betreten kann (Abb. 20). In den 1890er-Jahren als Gefängnis benutzt (Prison Boab), ist er heute eine touristische Attraktion.

\section{Sammlung australischer Pflanzen im ÖBG}

Der Ökologisch-Botanische Garten der Universität Bayreuth (ÖBG) beherbergt eine große und einzigartige Sammlung von Kalthauspflanzen (Kübelpflanzen) aus Australien, dem Mittelmeergebiet sowie den wärmeren Regionen Asiens, Afrikas und Amerikas. Gerade aus der australischen Flora kultiviert der ÖBG zahlreiche Arten, derzeit 50 Eucalyptus- (über 110 Individuen) und fünf Corymbia-Arten (11 Individuen). Die Exkursion nach Westaustralien bot die Gelegenheit, das australische Pflanzensortiment im ÖBG zu erweitern. Samen von etwa 60 Arten, von Acacia dunnii bis Xylomelum angustifolium, wurden mittlerweile ausgesät und werden hoffentlich gut gedeihen. Unter den aufgesammelten Arten, die nun im ÖBG in Kultur sind, waren 8 Corymbia- und 19 Eucalyptus-Arten, unter ihnen auch der erst 2009 von Dean Nicolle neu beschriebene Eucalyptus vittata (Abb. 8).

\section{Dank}

Unser besonderer Dank gilt Inge Schulze und Вов Nicolle, den guten Seelen und tatkräftigen (Not-)Helfern der Expedition.

Abb. 22 (oben): Blüte und junge Frucht von

Cochlospermum fraseri, einem Laub

abwerfenden Baum aus den Kimberleys.

Abb. 23 (Mitte): Blüten von Brachychiton fitzgeraldianus aus den Kimberleys.

Abb. 24 (unten): Blüten von Eucalyptus orbifolia - die sich streckenden Filamente der linken Blüte sprengen gerade das Operculum ab.
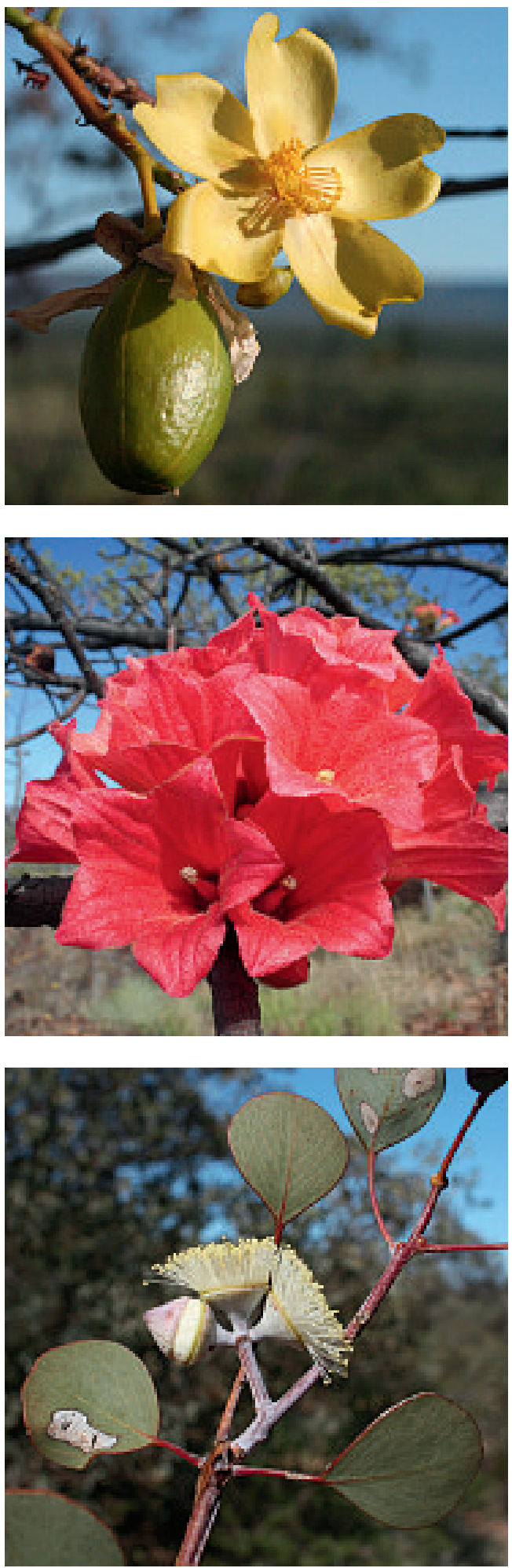


\section{Literatur}

Beadle, N. C. W. 1981: The vegetation of Australia. Stuttgart, New York.

Blakely, W. F. 1934: A key to the Eucalypts.

$1^{\text {st }}$ Edition. - Sydney.

Braun, B., Bernzen, A. \& Schüttemeyer, A. 2010:

Australien - ein Kontinent der Städte. Praxis Geographie 40: 4-9.

Brock, J. 2007: Native plants of northern Australia. New Holland.

Brooker, M. I. H. 2000: A new classification of the genus Eucalyptus L'HÉr. (Myrtaceae).

Austral. Syst. Bot. 13: 79-148.

Brooker M., I., H. \& Kleinig, D. A. 2006: Field guide to eucalypts. Volume 1, South-eastern Australia. $3^{\text {rd }}$ edition. - Melbourne.

Calladine, A \& Pate, J. 2000: Haustorial structure and functioning of the root hemiparasitic tree Nuytsia

floribunda (LABILL.) R.BR. and water relationships with its hosts. - Ann. Botany 85: 723-731.

Calladine A., Pate, J., \& Dixon, K. W. 2000: Haustorial development and growth benefit to seedlings of the root hemiparasitic tree Nuytsia floribunda (LABILL.) R.BR. in association with various hosts. - Ann. Botany 85: 733-740. Diels, L. 1906: Die Pflanzenwelt von West-Australien südlich des Wendekreises. - Leipzig.

Elliot, W. R. \& Jones, D. L. 1985: Encyclopedia of Australian plants suitable for cultivation. Vol 2.Melbourne, Sydney, Auckland.

Elliot, W. R. \& Jones, D. L. 1997: Encyclopedia of Australian plants suitable for cultivation. Vol 7. Melbourne, Sydney, Auckland.

Evenari, M., Shanan, L. \& Tadmor, N. 1982:

The Negev. The challenge of a desert. - Cambridge.

Fukarek F, Hübel, H., König, P., Müller, G. K., Schuster, R. \& Succow, M. 1995: Vegetation. - Leipzig, Jena, Berlin.

Hill, K. D. 1991: Eucalyptus. In: Harden G: Flora of New South Wales, Vol. 2. - NSW University Press. Hill, K. D. \& Johnson, L. A. S. 1995: Systematic studies in the eucalypts. 7. A revision of the bloodwoods, genus Corymbia (Myrtaceae). Telopea 6: 173-504.

Lowrie, A. (1987, 1989, 1998): Carnivorous plants of Australia. Vol 1-3. - Perth.

Lüpnitz, D. 1998: Gondwana. Die Pflanzenwelt von Australien und ihr Ursprung. Palmengarten Sonderheft.
LÜPNITZ, D. 2003: Australis. Lebensräume in Australien. Palmengarten Sonderheft Bd. 37.

Nicolle, D. 1997: Eucalypts of south Australia. - South Australia.

Pate, J. \& Dixon, K. 1982: Tuberous, comous and bulbous plants: biology of an adaptive strategy in western Australia. - Perth.

Pryor, L. D. \& Johnson, L. A. S. 1971: A classification of the eucalypts. - Canberra.

Schulze, E.-D., Gebauer, G., Schulze, W. \& Pate, J. S. 1991: The utilization of nitrogen from insect capture by different growth forms of Drosera from southwest Australia. Oecologia 87: 240-246.

Schulze E. D, Turner N.C., Nicolle, D. \& Schumacher, J. 2006 a: Leaf and wood carbon isotope ratios, specific leaf areas and wood growth of Eucalyptus species across a rainfall gradient in Australia. Tree Physiology 26: 479-492.

Schulze, E. D., Turner, N.C, Nicolle, D. \& Schumacher, J. 2006 b: Species differences in carbon isotope ratios, specific leaf area and nitrogen concentrations in leaves of Eucalyptus growing in a common garden compared with along an aridity gradient. Physiologia Plantarum 127: 434-444, doi 10.1111/j.1399-3054.2006.00682.x.

TAYlor, A. \& Hopper, D. 1991: The Banksia atlas. Australian flora and fauna series no 8. - Canberra. Thielke, T. 2010: Goldrausch in down under. Der Spiegel 33: 92-93.

Turner, N., Schulze, E. D, Nicolle, D. \& Kuhlmann, I. 2010: Growth in two common gardens reveals species by environment interactions in carbon isotope discrimination of Eucalyptus. Tree Physiology 30: 741-747, doi: 10.1093/ treephys/tpq029.

Westermann, Schroedel, Diesterweg 20io: Praxis Geographie, 40 (1), Karte aus dem Anhang. - Braunschweig.

Williams, J. A \& Woinarski, J. C. Z. 1997: Eucalypt ecology. Individuals to ecosystems. - Cambridge.

\section{Internetseiten}

http://www.theplantlist.org

http://florabase.calm.wa.gov.au/

http://en.wikipedia.org/wiki/Eucalyptus

http://www.dn.com.au/

http://www.obg.uni-bayreuth.de 